Pacific

Journal of

Mathematics

\title{
LANDAU-TOEPLITZ THEOREMS FOR SLICE REGULAR FUNCTIONS OVER QUATERNIONS
}

GRAZIANO GENTILI AND GiUlia SARFATti 


\title{
LANDAU-TOEPLITZ THEOREMS FOR SLICE REGULAR FUNCTIONS OVER QUATERNIONS
}

\author{
GraZiano Gentili AND GiUlia SARFATti
}

\begin{abstract}
The theory of slice regular functions of a quaternionic variable extends the notion of holomorphic function to the quaternionic setting. This theory, already rich in results, is sometimes surprisingly different from the theory of holomorphic functions of a complex variable; however, several fundamental results in the two environments are similar even if their proofs for the case of quaternions need new technical tools.

In this paper we prove the Landau-Toeplitz theorem for slice regular functions in a formulation that involves an appropriate notion of regular 2diameter. We show that the Landau-Toeplitz inequalities hold in the case of the regular $n$-diameter for all $n \geq 2$. Finally, a 3-diameter version of the Landau-Toeplitz theorem is proved using the notion of slice 3-diameter.
\end{abstract}

\section{Introduction}

The Schwarz lemma, in its different flavors, is the basis of a chapter of fundamental importance in the geometric theory of holomorphic functions of one and several complex variables. Its classic formulation in one variable is the following:

Theorem 1.1 (Schwarz lemma). Let $\mathbb{D}=\{z \in \mathbb{C}:|z|<1\}$ be the open unit disc of $\mathbb{C}$ centered at the origin, and let $f: \mathbb{D} \rightarrow \mathbb{D}$ be a holomorphic function such that $f(0)=0$. Then

$$
|f(z)| \leq|z|
$$

for all $z \in \mathbb{D}$, and

$$
\left|f^{\prime}(0)\right| \leq 1
$$

Equality holds in (1) for some $z \in \mathbb{D} \backslash\{0\}$, or in (2), if and only if there exists $u \in \mathbb{C}$ with $|u|=1$ such that $f(z)=u z$ for all $z \in \mathbb{D}$.

Gentili and Sarfatti were partially supported by GNSAGA of the INdAM and by PRIN "Proprietà geometriche delle varietà reali e complesse" of the MIUR. Sarfatti was partially supported by PRIN "Geometria differenziale e analisi globale" of the MIUR.

MSC2010: primary 30G35; secondary 30C80.

Keywords: functions of hypercomplex variables, geometric theory of regular functions of a quaternionic variable, Schwarz lemma and generalizations. 
The Schwarz lemma and its extension due to Pick lead in a natural way to the construction of the Poincaré metric, which plays a key role in the study of the hyperbolic geometry of complex domains and manifolds. In the same year of the first formulation of the Schwarz lemma, the Landau-Toeplitz theorem [1907] was proven. This less known but quite interesting result concerns the study of the possible shapes of the image of the unit disc under a holomorphic function and it is formulated in terms of the diameter of the image set.

Theorem 1.2 (Landau-Toeplitz [1907]; see also Burckel et al. 2006). Let $f$ be holomorphic in $\mathbb{D}$ and such that the diameter $\operatorname{diam} f(\mathbb{D})$ of $f(\mathbb{D})$ equals 2 . Then

$\operatorname{diam} f(r \mathbb{D}) \leq 2 r$

for all $r \in(0,1)$ and

$$
\left|f^{\prime}(0)\right| \leq 1 .
$$

Equality holds in (3) for some $r \in(0,1)$, or in (4), if and only if $f$ is of the form $f(z)=a+b z$, with $a, b \in \mathbb{C}$ and $|b|=1$.

This result can be interpreted as a generalization of the classical Schwarz lemma in which the diameter of the image set takes over the role of the maximum modulus of the function; indeed, there exist infinite subsets of the plane that have constant diameter and are different from a disc; the Reuleaux polygons are a well-known example of such sets [Gardner 2006; Lachand-Robert and Oudet 2007].

The recent definition of slice regularity for quaternionic functions of one quaternionic variable, inspired by Cullen [1965] and developed in [Gentili and Struppa 2006; 2007], identifies a large class of functions, which includes natural quaternionic power series and polynomials. The study of a geometric theory for this class of functions has by now produced several interesting results, sometimes analogous to those valid for holomorphic functions; the Schwarz lemma is among these results [Gentili and Struppa 2007], together with the Bohr theorem and the Bloch-Laudau theorem [Della Rocchetta et al. 2012; 2013; Sarfatti 2013].

Fairly new developments in the theory of holomorphic functions of one complex variable include the analogue of the Schwarz lemma for meromorphic functions, and open new fascinating perspectives for future research. In this setting, Solynin [2008] recalls into the scenery the approach of Landau and Toeplitz and its modern reinterpretation and generalization due to Burckel, Marshall, Minda, Poggi-Corradini and Ransford [Burckel et al. 2008].

In our paper, we first prove an analogue of the Landau-Toeplitz theorem for slice regular functions. To this purpose we need to introduce a new tool to "measure" the image of the open unit ball $\mathbb{B}$ of the space of quaternions $\mathbb{W}$ through a slice regular function, the regular diameter. 
Definition 1.3. Let $f$ be a slice regular function on $\mathbb{B}=\{q \in \mathbb{H}:|q|<1\}$ and let

$$
f(q)=\sum_{n \geq 0} q^{n} a_{n}
$$

be its power series expansion. For $r \in(0,1)$, we define the regular diameter of the image of $r \mathbb{B}$ under $f$ as

$$
\tilde{d}_{2}(f(r \mathbb{B}))=\max _{u, v \in \overline{\mathbb{B}}} \max _{|q| \leq r}\left|f_{u}(q)-f_{v}(q)\right|,
$$

where

$$
f_{u}(q)=\sum_{n \geq 0} q^{n} u^{n} a_{n} \quad \text { and } \quad f_{v}(q)=\sum_{n \geq 0} q^{n} v^{n} a_{n} .
$$

We define the regular diameter of the image of $\mathbb{B}$ under $f$ as

$$
\tilde{d}_{2}(f(\mathbb{B}))=\lim _{r \rightarrow 1^{-}} \tilde{d}_{2}(f(r \mathbb{B})) .
$$

The introduction of this new geometric quantity is necessary because of the peculiarities of the quaternionic environment, and in particular since a composition of slice regular functions is not slice regular in general. The regular diameter can play the role of the diameter; in fact, the former is finite if and only if the latter is finite. The regular diameter hence appears in the statement of the announced result. Theorem 3.9 (Landau-Toeplitz for regular functions). Let $f$ be a slice regular function on $\mathbb{B}$ such that $\tilde{d}_{2}(f(\mathbb{B}))=2$ and let $\partial_{c} f(0)$ be its slice derivative in 0 . Then

$$
\tilde{d}_{2}(f(r \mathbb{B})) \leq 2 r
$$

for all $r \in(0,1)$, and

$$
\left|\partial_{c} f(0)\right| \leq 1
$$

Equality holds in (5) for some $r \in(0,1)$, or in (6), if and only if $f$ is an affine function; that is, $f(q)=a+q b$ with $a, b \in \mathbb{U}$ and $|b|=1$.

As in the complex setting, this theorem can be interpreted as a generalization of the Schwarz lemma.

The new version of the Landau-Toeplitz theorem proposed in [Burckel et al. 2008] concerns holomorphic functions whose image is measured with a notion of diameter more general than the classic one, the $n$-diameter. In the quaternionic setting, the analogue of this geometric quantity is defined:

Definition 1.4. Let $E \subset \mathbb{H}$. For every $n \in \mathbb{N}, n \geq 2$, the $n$-diameter of $E$ is defined as

$$
d_{n}(E)=\sup _{w_{1}, \ldots, w_{n} \in E}\left(\prod_{1 \leq j<k \leq n}\left|w_{k}-w_{j}\right|\right)^{\frac{2}{n(n-1)}} .
$$


Retracing the approach used in the complex setting, we are able to obtain only the generalization of the first part of the statement of the Landau-Toeplitz theorem for the $n$-diameter. As in the case $n=2$, we need a notion of regular $n$-diameter $\tilde{d}_{n}(f(\mathbb{B}))$ for the image of $\mathbb{B}$ through a slice regular function $f$. This notion is a generalization of Definition 1.3 modeled on Definition 1.4 and given in terms of the $*$-product between slice regular functions (see Section 2). For all $n \geq 2$, the regular $n$-diameter turns out to be finite when the $n$-diameter is finite. For this reason, even if it may appear awkward, it makes sense to use the regular $n$-diameter in the following statement:

Theorem 1.5. Let $f$ be a slice regular function on $\mathbb{B}$ such that $\tilde{d}_{n}(f(\mathbb{B}))=d_{n}(\mathbb{B})$. Then

$$
\tilde{d}_{n}(f(r \mathbb{B})) \leq d_{n}(r \mathbb{B}) \quad \text { for all } r \in(0,1),
$$

and

$$
\left|\partial_{c} f(0)\right| \leq 1
$$

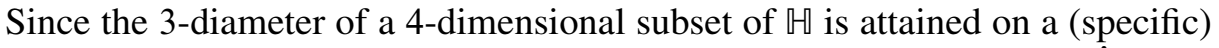
bidimensional section, we are encouraged to introduce an appropriate notion $\hat{d}_{3} f(\mathbb{B})$ of slice 3-diameter for $f(\mathbb{B})$ inspired by the power series expansion of the regular 3-diameter. This leads to the following complete result:

Theorem 5.7 (Landau-Toeplitz theorem for the slice 3-diameter). Let $f$ be a slice regular function on $\mathbb{B}$ such that $\hat{d}_{3}(f(\mathbb{B}))=d_{3}(\mathbb{B})$. Then

$$
\hat{d}_{3}(f(r \mathbb{B})) \leq d_{3}(r \mathbb{B})
$$

for every $r \in(0,1)$, and

$$
\left|\partial_{c} f(0)\right| \leq 1
$$

Equality holds in (7) for some $r \in(0,1)$, or in (8), if and only if $f$ is an affine function $f(q)=a+q b$ with $a, b \in \mathbb{H}$ and $|b|=1$.

We point out that all the extensions of the Landau-Toeplitz results presented in this paper generalize the Schwarz lemma to a much larger class of image sets; in fact, for all $n \geq 2$ there exist infinitely many subsets of the space $\mathbb{H}$ which have fixed $n$-diameter, do not coincide with a 4-ball, and neither contain nor are contained in the 4-ball. The 4-bodies of constant width are examples of such subsets, presented for instance in [Gardner 2006; Lachand-Robert and Oudet 2007].

\section{Preliminaries}

Let $\mathbb{H}$ be the skew field of quaternions obtained by endowing $\mathbb{R}^{4}$ with the multiplication operation defined on the standard basis $\{1, i, j, k\}$ by $i^{2}=j^{2}=$ $k^{2}=-1$ and $i j=k$, and then extended by distributivity to all quaternions 
$q=x_{0}+x_{1} i+x_{2} j+x_{3} k$. For every $q \in \mathbb{H}$, we define the real and imaginary part of $q$ as $\operatorname{Re} q=x_{0}$ and $\operatorname{Im} q=x_{1} i+x_{2} j+x_{3} k$, its conjugate as $\bar{q}=\operatorname{Re} q-\operatorname{Im} q$, and its modulus as $|q|^{2}=q \bar{q}$. The multiplicative inverse of $q \neq 0$ is $q^{-1}=\bar{q} /|q|^{2}$. Let $\mathbb{S}$ be the unit 2-sphere of purely imaginary quaternions, $\mathbb{S}=\left\{q \in \mathbb{M} \mid q^{2}=-1\right\}$. Then for any $I \in \mathbb{S}$, we will denote by $L_{I}$ the complex plane $\mathbb{R}+\mathbb{R} I$, and if $\Omega \subset \mathbb{H}$, we further set $\Omega_{I}=\Omega \cap L_{I}$. Notice that to every $q \in \mathbb{M} \backslash \mathbb{R}$, we can associate a unique element in $\mathbb{S}$ by the map $q \mapsto \operatorname{Im}(q) /|\operatorname{Im}(q)|=I_{q}$; therefore, for any $q \in \mathbb{U} \backslash \mathbb{R}$ there exist and are unique $x, y \in \mathbb{R}$ with $y>0$ and $I_{q} \in \mathbb{S}$ such that $q=x+y I_{q}$. If $q$ is real then $I_{q}$ can be any element of $\mathbb{S}$.

The preliminary results stated in this section will be given for slice regular functions defined on open balls of type $B=B(0, R)=\{q \in \mathbb{H}|| q \mid<R\}$. We point out that in most cases these results hold, with appropriate changes, for a more general class of domains introduced in [Colombo et al. 2009]. Let us now recall the definition of slice regularity.

Definition 2.1. A function $f: B=B(0, R) \rightarrow \mathbb{W}$ is said to be slice regular (often abbreviated to regular later on) if for every $I \in \mathbb{S}$, its restriction $f_{I}$ to $B_{I}$ has continuous partial derivatives and satisfies

$$
\bar{\partial}_{I} f(x+y I)=\frac{1}{2}\left(\frac{\partial}{\partial x}+I \frac{\partial}{\partial y}\right) f_{I}(x+y I)=0 \quad \text { for every } x+y I \in B_{I} .
$$

In the sequel we may refer to the vanishing of $\bar{\partial}_{I} f$ by saying that the restriction $f_{I}$ is holomorphic on $B_{I}$. In what follows, for the sake of shortness we will omit the prefix slice when referring to slice regular functions. A notion of derivative, called slice (or Cullen) derivative, can be given for regular functions by

$$
\partial_{c} f(x+y I)=\frac{\partial}{\partial x} f(x+y I) \quad \text { for } x+y I \in B .
$$

This definition is well-posed because it is applied only to regular functions; moreover, slice regularity is preserved by slice differentiation. A basic result connects slice regularity and classical holomorphy:

Lemma 2.2 (splitting lemma; see [Gentili and Struppa 2007]). If $f$ is a regular function on $B=B(0, R)$ then for every $I \in \mathbb{S}$ and for every $J \in \mathbb{S}$ with $J$ orthogonal to $I$, there exist two holomorphic functions $F, G: B_{I} \rightarrow L_{I}$ such that

$$
f_{I}(z)=F(z)+G(z) J \text { for every } z=x+y I \in B_{I} .
$$

Theorem 2.3 [Gentili and Struppa 2007]. A function $f$ is regular on $B=B(0, R)$ if and only if $f$ has a power series expansion

$$
f(q)=\sum_{n \geq 0} q^{n} a_{n} \quad \text { with } \quad a_{n}=\frac{1}{n !} \frac{\partial^{n} f}{\partial x^{n}}(0)
$$

converging absolutely and uniformly on compact sets in $B(0, R)$. 
The next two results will be needed later.

Theorem 2.4 (identity principle, weak version; see [Gentili and Struppa 2007]). Let $f: B=B(0, R) \rightarrow \mathbb{W}$ be a regular function. Denote by $Z_{f}$ the zero set of $f, Z_{f}=\{q \in B \mid f(q)=0\}$. If there exists $I \in \mathbb{S}$ such that $B_{I} \cap Z_{f}$ has an accumulation point in $B_{I}$ then $f$ vanishes identically on $B$.

Theorem 2.5 (representation formula; see [Colombo et al. 2009]). Let $f$ be a regular function on $B=B(0, R)$ and let $J \in \mathbb{S}$. Then for all $x+y I \in B$, the following equality holds:

$$
f(x+y I)=\frac{1}{2}[f(x+y J)+f(x-y J)]+I \frac{1}{2}[J(f(x-y J)-f(x+y J))] .
$$

The product of two regular functions is not, in general, regular. To guarantee regularity we need to introduce the following multiplication operation:

Definition 2.6. Let $f(q)=\sum_{n \geq 0} q^{n} a_{n}$ and $g(q)=\sum_{n \geq 0} q^{n} b_{n}$ be regular functions on $B=B(0, R)$. The $*$-product of $f$ and $g$ is the regular function

$$
f * g: B \rightarrow \mathbb{W}
$$

defined by

$$
f * g(q)=\sum_{n \geq 0} q^{n} \sum_{k=0}^{n} a_{k} b_{n-k} .
$$

The $*$-product is associative but not, in general, commutative. The following result clarifies the relation between the $*$-product and the pointwise product of regular functions.

Proposition 2.7 [Gentili et al. 2013]. Let $f(q)=\sum_{n \geq 0} q^{n} a_{n}$ and $g(q)=\sum_{n \geq 0} q^{n} b_{n}$
be regular functions on $B=B(0, R)$. Then

$$
f * g(q)= \begin{cases}f(q) g\left(f(q)^{-1} q f(q)\right) & \text { if } f(q) \neq 0, \\ 0 & \text { if } f(q)=0 .\end{cases}
$$

Notice that if $q=x+y I$ (and if $f(q) \neq 0)$ then $f(q)^{-1} q f(q)$ has the same modulus and same real part as $q$; hence, $T_{f}(q)=f(q)^{-1} q f(q)$ lies in $x+y \mathbb{S}$, the same 2-sphere as $q$. A zero $x_{0}+y_{0} I$ of the function $g$ is not necessarily a zero of $f * g$, but one element on the same sphere $x_{0}+y_{0} \mathbb{S}$ is.

To conclude this section we recall a result that is basic for our purposes.

Theorem 2.8 (maximum modulus principle [Gentili and Struppa 2007]). Let $f$ : $B \rightarrow \mathbb{W}$ be a regular function. If there exists $I \in \mathbb{S}$ such that the restriction $\left|f_{I}\right|$ has a local maximum in $B_{I}$ then $f$ is constant in $B$. In particular, if $|f|$ has a local maximum in $B$ then $f$ is constant in $B$. 


\section{The Landau-Toeplitz theorem for regular functions}

In this section, we will prove our analogue of the Landau-Toeplitz theorem for (quaternionic) regular functions. To reach the aim, we will need a few steps.

Denote by $\langle$,$\rangle the scalar product of \mathbb{R}^{4}$, and by $\times$ the vector product of $\mathbb{R}^{3}$. Recall the equality $u v=-\langle u, v\rangle+u \times v$, valid for purely imaginary quaternions $u, v$. Also, if $w=x+y L \in \mathbb{U}$, then $\langle w, I\rangle=\langle y L, I\rangle=-\operatorname{Re}(y L I)=-\operatorname{Re}(w I)$ for all $I \in \mathbb{S}$.

Definition 3.1. Let $I \in \mathbb{S}$. For any $w \in \mathbb{U}$, we define the imaginary component of $w$ along $I$ as $\operatorname{Im}_{I}(w)=\langle w, I\rangle=-\operatorname{Re}(w I)$.

Proposition 3.2. Let $w \in B=B(0, R), 0<|w|=r<R$, and let $g$ be a holomorphic function on $B \cap L_{I_{w}}$. If

$$
g(w)=w \quad \text { and } \quad r=\max _{z \in r \overline{\mathbb{B}}_{I w}}|g(z)|
$$

then $\operatorname{Im}_{I_{w}}\left(\partial_{c} g(w)\right)=0$.

Proof. To simplify the notation, set $I=I_{w}$. Define $\varphi: \mathbb{R} \rightarrow \mathbb{R}$ by $\varphi(\theta)=\left|g\left(w e^{I \theta}\right)\right|^{2}$. The splitting lemma (2.2) implies that for every $J \in \mathbb{S}$ orthogonal to $I$ there exist holomorphic functions $F, G: B_{I} \rightarrow L_{I}$ such that $g(z)=F(z)+G(z) J$ for every $z \in B_{I}$. A direct computation shows that $\varphi(\theta)=F\left(w e^{I \theta}\right) \overline{F\left(w e^{I \theta}\right)}+$ $G\left(w e^{I \theta}\right) \overline{G\left(w e^{I \theta}\right)}$; hence,

$$
\varphi^{\prime}(\theta)=-2 \operatorname{Im}_{I}\left(w e^{I \theta}\left(F^{\prime}\left(w e^{I \theta}\right) \overline{F\left(w e^{I \theta}\right)}+G^{\prime}\left(w e^{I \theta}\right) \overline{G\left(w e^{I \theta}\right)}\right)\right),
$$

where $F^{\prime}$ and $G^{\prime}$ are the complex derivatives of $F$ and $G$ in $B_{I}$. Since, by hypothesis, $\theta=0$ is a maximum for $\varphi$, we have

$$
0=\varphi^{\prime}(0)=-2 \operatorname{Im}_{I}\left(w\left(F^{\prime}(w) \overline{F(w)}+G^{\prime}(w) \overline{G(w)}\right)\right) .
$$

Moreover, $w=g(w)=F(w)+G(w) J$, which implies $F(w)=w$ and $G(w)=0$. Putting these values in (10), we have $0=-2 \operatorname{Im}_{I}\left(w F^{\prime}(w) \bar{w}\right)=-2|w|^{2} \operatorname{Im}_{I}\left(F^{\prime}(w)\right)$, which yields $\operatorname{Im}_{I}\left(F^{\prime}(w)\right)=0$. Finally, recalling the definition of the slice derivative and Definition 3.1, we get

$$
\operatorname{Im}_{I}\left(\partial_{c} g(w)\right)=\operatorname{Im}_{I}\left(F^{\prime}(w)+G^{\prime}(w) J\right)=\operatorname{Im}_{I}\left(F^{\prime}(w)\right)=0 .
$$

Remark 3.3. The proposition can be interpreted as a consequence of the JuliaWolff-Carathéodory theorem (see for instance [Abate 1989; Burckel 1979]); in fact, the hypotheses in (9) yield that $g: r \mathbb{B}_{I} \rightarrow r \mathbb{B}$ and that $w$ is a boundary fixed point for the restriction of $g$ to $r \mathbb{B}_{I}$; hence, if we split the function $g$ as $g(z)=F(z)+G(z) J$, for $z \in r \mathbb{B}_{I}$, we have that $w$ is a Wolff point for $F: r \mathbb{B}_{I} \rightarrow r \mathbb{B}_{I}$. 
The proof of the classical Landau-Toeplitz theorem in the setting of holomorphic maps [Burckel et al. 2008] relies upon the analogue of Proposition 3.2, which is not sufficient for our purposes in the quaternionic environment; in fact, we need the following:

Proposition 3.4. Let $g: \mathbb{B} \rightarrow \mathbb{H}$ be a regular function such that $\operatorname{Im}_{I_{q}}(g(q))=0$ for every $q \in \mathbb{B}$. Then $g$ is a real constant function.

Proof. Let $g(q)=\sum_{n \geq 0} q^{n} a_{n}$ on $\mathbb{B}$. For any $I \in \mathbb{S}$, we split the coefficient $a_{n}$ as $b_{n}+c_{n} J$ with $b_{n}, c_{n} \in L_{I}$ and $J \in \mathbb{S}$ orthogonal to $I$. By hypothesis, we have

$$
0=\operatorname{Im}_{I}(g(z))=\operatorname{Im}_{I}\left(\sum_{n \geq 0} z^{n}\left(b_{n}+c_{n} J\right)\right)=\operatorname{Im}_{I}\left(\sum_{n \geq 0} z^{n} b_{n}\right) \text { for all } z \in \mathbb{B}_{I} .
$$

As a consequence of the open mapping theorem, the holomorphic map $\sum_{n \geq 0} z^{n} b_{n}$ is constant; that is, $b_{n}=0$ for all $n>0$. Therefore, the component of each $a_{n}$ along $L_{I}$ vanishes for all $n>0$. Since $I \in \mathbb{S}$ is arbitrary, this implies $a_{n}=0$ for all $n>0$. The hypothesis yields that $a_{0} \in \mathbb{R}$.

A basic notion used to state the classical Landau-Toeplitz theorem is the diameter of the images of holomorphic functions. In the new quaternionic setting, due to the fact that composition of regular functions is not regular in general, the definition of a "regular" diameter for the images of regular functions requires a peculiar approach.

Definition 3.5. Let $f: \mathbb{B} \rightarrow \mathbb{W}$ be a regular function $f(q)=\sum_{n \geq 0} q^{n} a_{n}$, and let $u \in \mathbb{H}$. We define the regular composition of $f$ with the function $q \mapsto q u$ as

$$
f_{u}(q)=\sum_{n \geq 0}(q u)^{* n} a_{n}=\sum_{n \geq 0} q^{n} u^{n} a_{n} .
$$

If $|u|=1$, the radius of convergence of the series expansion for $f_{u}$ is the same as that for $f$. Moreover, if $u$ and $q_{0}$ lie in the same plane $L_{I}$ then $u$ and $q_{0}$ commute, hence $f_{u}\left(q_{0}\right)=f\left(q_{0} u\right)$. In particular, if $u \in \mathbb{R}$ then $f_{u}(q)=f(q u)$ for every $q$.

Definition 3.6. Let $f: \mathbb{B} \rightarrow \mathbb{H}$ be a regular function. For $r \in(0,1)$, we define the regular diameter of the image of $r \mathbb{B}$ under $f$ as

$$
\tilde{d}_{2}(f(r \mathbb{B}))=\max _{u, v \in \overline{\mathbb{B}}} \max _{|q| \leq r}\left|f_{u}(q)-f_{v}(q)\right| .
$$

We define the regular diameter of the image of $\mathbb{B}$ under $f$ as

$$
\tilde{d}_{2}(f(\mathbb{B}))=\lim _{r \rightarrow 1^{-}} \tilde{d}_{2}(f(r \mathbb{B})) .
$$

Remark 3.7. By the maximum modulus principle for regular functions, $\tilde{d}_{2}(f(r \mathbb{B}))$ is an increasing function of $r$; hence the limit (11) always exists. So $\tilde{d}_{2}(f(\mathbb{B}))$ is well defined. 
Let $E$ be a subset of $\mathbb{H}$. We will denote by $\operatorname{diam} E=\sup _{q, w \in E}|q-w|$ the classical diameter of $E$.

Proposition 3.8. Let $f$ be a regular function on $\mathbb{B}$. Then

$$
\operatorname{diam} f(\mathbb{B}) \leq \tilde{d}_{2}(f(\mathbb{B})) \leq 2 \operatorname{diam} f(\mathbb{B}) .
$$

Proof. To prove the first inequality, let $r \in(0,1)$ and consider $q, w \in r \overline{\mathbb{B}}$. We want to bound $|f(q)-f(w)|$. Suppose without loss of generality that $|w| \geq|q|$ and $w \neq 0$. Then

$$
|f(q)-f(w)|=\left|f\left(q \frac{|w|}{|w|}\right)-f\left(w \frac{|w|}{|w|}\right)\right|=\left|f_{\frac{q}{|w|}}(|w|)-f_{\frac{w}{|w|}}(|w|)\right|
$$

where the last equality is due to the fact that $|w|$, being real, commutes with both $q /|w|$ and $w /|w|$. Since $q /|w| \in \overline{\mathbb{B}}$ and $w /|w| \in \partial \mathbb{B},(12)$ yields

$$
\begin{aligned}
|f(q)-f(w)| & \leq \max _{u, v \in \overline{\mathbb{B}}}\left|f_{u}(|w|)-f_{v}(|w|)\right| \\
& \leq \max _{u, v \in \overline{\mathbb{B}}} \max _{|q| \leq r}\left|f_{u}(q)-f_{v}(q)\right|=\tilde{d}_{2}(f(r \mathbb{B})) .
\end{aligned}
$$

This implies that diam $f(r \overline{\mathbb{B}}) \leq \tilde{d}_{2}(f(r \mathbb{B}))$. Since this inequality holds for any $r \in(0,1)$, we obtain

$$
\operatorname{diam} f(\mathbb{B})=\lim _{r \rightarrow 1^{-}} \operatorname{diam} f(r \overline{\mathbb{B}}) \leq \lim _{r \rightarrow 1^{-}} \tilde{d}_{2}(f(r \mathbb{B}))=\tilde{d}_{2}(f(\mathbb{B})) .
$$

To show the missing inequality, let $u, v \in \overline{\mathbb{B}}, r \in(0,1)$, and let $J, K$ be elements of $\mathbb{S}$ such that $u \in L_{J}$ and $v \in L_{K}$. Using the representation formula (see Theorem 2.5) and taking into account that $u$ and $x+y J$ commute as well as $v$ and $x+y K$, we get, for all $q=x+y I \in r \overline{\mathbb{B}}$,

$$
\begin{aligned}
& \left|f_{u}(q)-f_{v}(q)\right| \\
& =\frac{1}{2} \mid(f((x+y J) u)-(f(x+y K) v))+(f((x-y J) u)-f((x-y K) v)) \\
& \quad+I J(f((x-y J) u)-f((x+y J) u))-I K(f((x-y K) v)-f((x+y K) v)) \mid \\
& \leq \frac{1}{2}|f((x+y J) u)-(f(x+y K) v)|+\frac{1}{2}|f((x-y J) u)-f((x-y K) v)| \\
& \quad+\frac{1}{2}|f((x-y J) u)-f((x+y J) u)|+\frac{1}{2}|f((x-y K) v)-f((x+y K) v)| \\
& \leq 2 \operatorname{diam} f(r \mathbb{B}) .
\end{aligned}
$$

Since inequality (13) holds for every $u, v \in \overline{\mathbb{B}}$ and for every $q \in r \overline{\mathbb{B}}$, we get

$$
\tilde{d}_{2}(f(r \mathbb{B}))=\max _{u, v \in \overline{\mathbb{B}}|q| \leq r} \max _{u}\left|f_{u}(q)-f_{v}(q)\right| \leq 2 \operatorname{diam} f(r \mathbb{B})
$$

and since this holds for every $r \in(0,1)$, we get $\tilde{d}_{2}(f(\mathbb{B})) \leq 2 \operatorname{diam} f(\mathbb{B})$. 
Notice that if $f$ is an affine function, say $f(q)=a+q b$, then for every $r \in(0,1)$ we have $\tilde{d}_{2}(f(r \mathbb{B}))=|b| \operatorname{diam} r \mathbb{B}=|b| r$ diam $\mathbb{B}$. In particular, if $f$ is constant then $\tilde{d}_{2}(f(r \mathbb{B}))=0$. Moreover, the regular diameter $\tilde{d}_{2}(f(r \mathbb{B}))$ is invariant under translations; in fact, if $g(q)=f(q)-f(0)$ then $\tilde{d}_{2}(g(r \mathbb{B}))=\tilde{d}_{2}(f(r \mathbb{B}))$ for every $r \in(0,1)$.

Theorem 3.9 (Landau-Toeplitz for regular functions). Let $f: \mathbb{B} \rightarrow \mathbb{W}$ be a regular function such that $\tilde{d}_{2}(f(\mathbb{B}))=\operatorname{diam} \mathbb{B}=2$. Then

$$
\tilde{d}_{2}(f(r \mathbb{B})) \leq 2 r
$$

for every $r \in(0,1)$, and

$$
\left|\partial_{c} f(0)\right| \leq 1
$$

Equality holds in (15) for some $r \in(0,1)$, or in (16), if and only if $f$ is an affine function $f(q)=a+q b$ with $a, b \in \mathbb{W}$ and $|b|=1$.

Proof. To prove the first inequality, take $u, v \in \overline{\mathbb{B}}$ and consider the auxiliary function

$$
g_{u, v}(q)=\frac{1}{2} q^{-1}\left(f_{u}(q)-f_{v}(q)\right) .
$$

This function is regular on $\mathbb{B}$; indeed, if the power series expansion of $f$ in $\mathbb{B}$ is $\sum_{n \geq 0} q^{n} a_{n}$, then

$$
g_{u, v}(q)=\frac{1}{2} q^{-1}\left(\sum_{n \geq 0} q^{n} u^{n} a_{n}-\sum_{n \geq 0} q^{n} v^{n} a_{n}\right)=\frac{1}{2} \sum_{n \geq 0} q^{n}\left(u^{n+1}-v^{n+1}\right) a_{n+1} .
$$

From this expression of $g_{u, v}$ we can recover its value at $q=0$ :

$$
g_{u, v}(0)=\frac{1}{2}(u-v) a_{1}=\frac{1}{2}(u-v) \partial_{c} f(0) .
$$

Since $g_{u, v}$ is a regular function, using the maximum modulus principle we get that

$$
r \mapsto \max _{u, v \in \overline{\mathbb{B}}} \max _{|q| \leq r}\left|g_{u, v}(q)\right|
$$

is increasing on $(0,1)$. Moreover, the regularity of the function $q \mapsto f_{u}(q)-f_{v}(q)$ yields that for any fixed $r \in(0,1)$ we can write

$$
\max _{|q| \leq r}\left|g_{u, v}(q)\right|=\max _{|q| \leq r} \frac{\left|f_{u}(q)-f_{v}(q)\right|}{2|q|}=\frac{1}{2 r} \max _{|q| \leq r}\left|f_{u}(q)-f_{v}(q)\right|,
$$

which leads to

$$
\frac{\tilde{d}_{2}(f(r \mathbb{B}))}{2 r}=\frac{1}{2 r} \max _{u, v \in \mathbb{B}} \max _{|q| \leq r}\left|f_{u}(q)-f_{v}(q)\right|=\max _{u, v \in \mathbb{B}} \max _{|q| \leq r}\left|g_{u, v}(q)\right| ;
$$

therefore, $\tilde{d}_{2}(f(r \mathbb{B})) / 2 r$ is an increasing function of $r$ and so always less than or 
equal to the limit

$$
\lim _{r \rightarrow 1^{-}} \frac{1}{2 r} \tilde{d}_{2}(f(r \mathbb{B}))=\frac{1}{2} \tilde{d}_{2}(f(\mathbb{B}))=1 .
$$

This means that

$$
\tilde{d}_{2}(f(r \mathbb{B})) \leq 2 r \text { for every } r \in(0,1),
$$

proving inequality (15). To prove (16), consider the odd part of $f$,

$$
f_{\text {odd }}(q)=\frac{f(q)-f(-q)}{2} .
$$

It satisfies the hypotheses of the Schwarz lemma for regular functions (see [Gentili and Struppa 2007]); indeed, $f_{\text {odd }}$ is a regular function on $\mathbb{B}, f_{\text {odd }}(0)=0$, and

$$
\left|f_{\text {odd }}(q)\right|=\frac{1}{2}|f(q)-f(-q)| \leq \frac{1}{2} \tilde{d}_{2}(f(\mathbb{B}))=1 \quad \text { for every } q \in \mathbb{B} ;
$$

hence,

$$
\begin{aligned}
1 \geq\left|\partial_{c} f_{\text {odd }}(0)\right| & =\left.\frac{1}{2}\left|\partial_{c} f(q)-\partial_{c}(f(-q))\right|\right|_{q=0} \\
& =\left.\frac{1}{2}\left|\partial_{c} f(q)+\partial_{c} f(-q)\right|\right|_{q=0}=\left|\partial_{c} f(0)\right| .
\end{aligned}
$$

We will now prove the last part of the statement, covering the case of equality. To begin with, notice that if $f(q)=a+q b$ with $a, b \in \mathbb{U}$ and $|b|=1$, then equality holds in both (15) and (16).

Conversely, suppose that equality holds in (16), so $\left|\partial_{c} f(0)\right|=1$. In this case we have $\left|\partial_{c} f_{\text {odd }}(0)\right|=1$; therefore, by the Schwarz lemma (see [Gentili and Struppa 2007]),

$$
f_{\text {odd }}(q)=q \partial_{c} f(0) \text {. }
$$

We want to show that in this case $\tilde{d}_{2}(f(r \mathbb{B}))=2 r$ for every $r \in(0,1)$; in fact, from (17) and (18) it follows that

$$
\frac{\tilde{d}_{2}(f(r \mathbb{B}))}{2 r} \geq \max _{u, v \in \mathbb{B}}\left|g_{u, v}(0)\right|=\max _{u, v \in \overline{\mathbb{B}}} \frac{1}{2}\left|(u-v) \partial_{c} f(0)\right|=1 \quad \text { for every } r \in(0,1) .
$$

Comparing the last inequality with (19) we get

$$
\tilde{d}_{2}(f(r \mathbb{B}))=2 r \quad \text { for every } r \in(0,1) .
$$

We now introducd a new auxiliary function. Take $w \in \mathbb{B}$ with $0<|w|=r<1$ and set

$$
h_{w}(q)=\frac{1}{2}(f(q)-f(-w)) \partial_{c} f(0)^{-1} .
$$

The function $h_{w}$ is regular on $\mathbb{B}$ and fixes $w$; indeed,

$$
h_{w}(w)=\frac{1}{2}(f(w)-f(-w)) \partial_{c} f(0)^{-1}=f_{\text {odd }}(w) \partial_{c} f(0)^{-1}=w,
$$


where the last equality is due to (21). We need now to restrict our attention to what happens in $L_{I_{w}}$. By the maximum modulus principle (Theorem 2.8), we are able to find $z_{0} \in L_{I_{w}},\left|z_{0}\right|=r$ such that for $z \in L_{I_{w}}$,

$$
\max _{|z| \leq r}\left|h_{w}(z)\right|=\frac{1}{2} \max _{|z| \leq r}|f(z)-f(-w)|=\frac{1}{2}\left|f\left(z_{0}\right)-f(-w)\right| .
$$

Let $\hat{u} \in L_{I_{w}}$ with $|\hat{u}|=1$ be such that $-w=z_{0} \hat{u}$. Then again for $z \in L_{I_{w}}$, due to the fact that $z_{0}$ and $\hat{u}$ commute,

$$
\begin{aligned}
\max _{|z| \leq r}\left|h_{w}(z)\right| & =\frac{1}{2}\left|f\left(z_{0}\right)-f\left(z_{0} \hat{u}\right)\right| \\
& =\frac{1}{2}\left|f\left(z_{0}\right)-f_{\hat{u}}\left(z_{0}\right)\right| \leq \frac{1}{2} \max _{u, v \in \mathbb{B}} \max _{|z| \leq r}\left|f_{u}(z)-f_{v}(z)\right| .
\end{aligned}
$$

Recalling (22) for $z \in L_{I_{w}}$ and $q \in \mathbb{W}$ we obtain

$$
\max _{|z| \leq r}\left|h_{w}(z)\right| \leq \frac{1}{2} \max _{u, v \in \overline{\mathbb{B}}} \max _{|q| \leq r}\left|f_{u}(q)-f_{v}(q)\right|=\frac{1}{2} \tilde{d}_{2}(f(r \mathbb{B}))=r=\left|h_{w}(w)\right| .
$$

The function $h_{w}$ then satisfies the hypotheses of Proposition 3.2; hence,

$$
0=\operatorname{Im}_{I_{w}}\left(\left.\partial_{c} h_{w}(q)\right|_{q=w}\right)=\operatorname{Im}_{I_{w}}\left(\frac{1}{2} \partial_{c} f(w) \partial_{c} f(0)^{-1}\right) .
$$

Now recall that $w$ is an arbitrary element of $\mathbb{B} \backslash\{0\}$. By continuity, we get that the function $w \mapsto \frac{1}{2} \partial_{c} f(w) \partial_{c} f(0)^{-1}$, regular on $\mathbb{B}$, satisfies the hypotheses of Proposition 3.4. Consequently, $\frac{1}{2} \partial_{c} f(w) \partial_{c} f(0)^{-1}$ is a real constant function hence $\partial_{c} f(w)$ is constant as well; therefore, $f$ has the required form $f(q)=$ $f(0)+q \partial_{c} f(0)$.

We will show now how equality in (15) for some $s \in(0,1)$ implies equality in (16); this and the preceding step will conclude the proof. Suppose that there exists $s \in(0,1)$ such that $\tilde{d}_{2}(f(s \mathbb{B})) / 2 s=1$. By (19) and since $\tilde{d}_{2}(f(r \mathbb{B})) / 2 r$ is increasing in $r$, we have

$$
\frac{\tilde{d}_{2}(f(r \mathbb{B}))}{2 r}=1 \quad \text { for every } r \in[s, 1) .
$$

Let us prove that this equality holds for all $r \in(0,1)$. Let $\hat{u}, \hat{v} \in \overline{\mathbb{B}}$ be such that

$$
\frac{\tilde{d}_{2}(f(s \mathbb{B}))}{2 s}=\max _{u, v \in \mathbb{B}} \max _{|q| \leq s}\left|g_{u, v}(q)\right|=\max _{|q| \leq s}\left|g_{\hat{u}, \hat{v}}(q)\right|,
$$

where the first equality follows from (18). Let $r>s$. By the choice of $\hat{u}, \hat{v} \in \overline{\mathbb{B}}$, we get

$$
1=\frac{\tilde{d}_{2}(f(r \mathbb{B}))}{2 r}=\max _{u, v \in \mathbb{B}} \max _{|q| \leq r}\left|g_{u, v}(q)\right| \geq \max _{|q| \leq r}\left|g_{\hat{u}, \hat{v}}(q)\right| \geq \max _{|q| \leq s}\left|g_{\hat{u}, \hat{v}}(q)\right|=1 .
$$


By the maximum modulus principle, the function $g_{\hat{u}, \hat{v}}$ must be constant in $q \in \mathbb{B}$ and equal to 1 in modulus. Now consider $r \in(0, s)$. Then

$$
1 \geq \frac{\tilde{d}_{2}(f(r \mathbb{B}))}{2 r}=\max _{u, v \in \mathbb{B}} \max _{|q| \leq r}\left|g_{u, v}(q)\right| \geq \max _{|q| \leq r}\left|g_{\hat{u}, \hat{v}}(q)\right|=1,
$$

which implies $\tilde{d}_{2}(f(r \mathbb{B})) / 2 r=1$ for every $r \in(0,1)$. The claim is now that $\left|\partial_{c} f(0)\right|=1$. By (20), we first of all obtain

$$
\lim _{r \rightarrow 0^{+}} \frac{\tilde{d}_{2}(f(r \mathbb{B}))}{2 r}=1 \geq\left|\partial_{c} f(0)\right| .
$$

Recalling that

$$
\frac{\tilde{d}_{2}(f(r \mathbb{B}))}{2 r}=\max _{u, v \in \overline{\mathbb{B}}} \max _{|q| \leq r}\left|g_{u, v}(q)\right|,
$$

we can get, for every $n \in \mathbb{N}$, the existence of $u_{n}, v_{n} \in \overline{\mathbb{B}}$ and $q_{n}$ with $\left|q_{n}\right|=\frac{1}{n}$ (converging up to subsequences), such that

$$
1=\lim _{n \rightarrow \infty} \frac{\tilde{d}_{2}\left(f\left(\frac{1}{n} \mathbb{B}\right)\right)}{2 \frac{1}{n}}=\lim _{n \rightarrow \infty}\left|g_{u_{n}, v_{n}}\left(q_{n}\right)\right|=\left|g_{\tilde{u}, \tilde{v}}(0)\right| \leq \max _{u, v \in \mathbb{B}}\left|g_{u, v}(0)\right|=\left|\partial_{c} f(0)\right| .
$$

(The last equality is due to (17).) A comparison with (23) concludes the proof.

\section{The $n$-diameter case}

We next prove the $n$-diameter version of the Landau-Toeplitz theorem for regular functions. Recall from Definition 1.4 the definition of the $n$-diameter of a subset of $\mathbb{H}$. As in the complex case (see [Burckel et al. 2008]), we have:

Proposition 4.1. For all $n \geq 2$, we have $d_{n}(E) \leq d_{2}(E)=$ diam $E$. Moreover, $d_{n}(E)$ is finite if and only if $d_{2}(E)$ is finite.

As we did in Section 3, in the case of the classical diameter $d_{2}$, we will adopt a specific definition for the $n$-diameter of the image of a subset of $\mathbb{W}$ under a regular function. We will always consider images of open balls of the form $r \mathbb{B}$.

Definition 4.2. Let $n \geq 2$ and let $f$ be a regular function on $\mathbb{B}$. For $r \in(0,1)$ we define, in terms of the $*$-product, the regular $n$-diameter of the image of $r \mathbb{B}$ under $f$ as

$$
\tilde{d}_{n}(f(r \mathbb{B}))=\max _{w_{1}, \ldots, w_{n} \in \mathbb{B}} \max _{|q| \leq r}\left|\prod_{1 \leq j<k \leq n}\left(f_{w_{k}}(q)-f_{w_{j}}(q)\right)\right|^{\frac{2}{n(n-1)}} .
$$

We define the regular $n$-diameter of the image of $\mathbb{B}$ under $f$ as

$$
\tilde{d}_{n}(f(\mathbb{B}))=\lim _{r \rightarrow 1^{-}} \tilde{d}_{n}(f(r \mathbb{B})) .
$$


The same argument used for the regular diameter in Remark 3.7 guarantees that $\tilde{d}_{n}(f(\mathbb{B}))$ is well-defined. Notice that because of the noncommutativity of quaternions, the order of the factors of a $*$-product has its importance. We can choose any order we like, but it has to be fixed once chosen. In what follows, when we write $1 \leq j<k \leq n$ we always mean to order the couples $(j, k)$ with the lexicographic order. To simplify the notation, we will sometimes write $j<k$, meaning $1 \leq j<k \leq n$.

The first step toward understanding the relation between the $n$-diameter and the regular $n$-diameter is the following result:

Proposition 4.3. Let $f: \mathbb{B} \rightarrow \mathbb{H}$ be a regular function, and let $n \geq 2$. Then $\tilde{d}_{n}(f(\mathbb{B})) \leq \tilde{d}_{2}(f(\mathbb{B}))$.

Proof. We omit the (technical) proof. The idea is to turn the $*$-product into a usual product with an iterated application of Proposition 2.7.

Notice that Proposition 4.1 and Proposition 4.3 imply that if $d_{n}(f(\mathbb{B}))$ is finite then $\tilde{d}_{n}(f(\mathbb{B}))$ is finite as well (for any regular function $f$ and $n \geq 2$ ).

Let us make some simple remarks about the definition of regular $n$-diameter. As for the case $n=2$, the regular $n$-diameter is invariant under translation; in fact, if $f$ is a regular function on $\mathbb{B}$ and $g$ is defined as $g(q)=f(q)-f(0)$ then $\tilde{d}_{n}(g(r \mathbb{B}))=\tilde{d}_{n}(f(r \mathbb{B}))$. Moreover, if $f(q)=q b$ with $b \in \mathbb{H}$ then $\tilde{d}_{n}(f(r \mathbb{B}))=$ $|b| d_{n}(r \mathbb{B})$. In particular, if $f$ is constant then $\tilde{d}_{n}(f(r \mathbb{B}))=0$; hence, if $f$ is of the form $f(q)=a+q b$ for some quaternions $a$ and $b$ then the regular $n$-diameter of $f(r \mathbb{B})$ coincides with its $n$-diameter.

In order to obtain analogues of inequalities (15) and (16) in the $n$-diameter case, we study the ratio between the regular $n$-diameter of the image of $r \mathbb{B}$ under a regular function $f$ and the $n$-diameter of the domain $r \mathbb{B}$ of $f$.

Lemma 4.4. Let $f$ be a regular function on $\mathbb{B}$ and let $n \in \mathbb{N}, n \geq 2$. Then

$$
\varphi_{n}(r)=\frac{\tilde{d}_{n}(f(r \mathbb{B}))}{d_{n}(r \mathbb{B})}=\frac{\tilde{d}_{n}(f(r \mathbb{B}))}{d_{n}(\mathbb{B}) r}
$$

is an increasing function of $r$ on the open interval $(0,1)$, and

$$
\lim _{r \rightarrow 0^{+}} \varphi_{n}(r)=\left|\partial_{c} f(0)\right| .
$$

Proof. If $f$ is a constant or affine function, $\varphi_{n}(r)$ is a constant function. So let $f$ be neither constant nor affine. Fix $w_{1}, \ldots, w_{n} \in \overline{\mathbb{B}}$ and consider the auxiliary function

$$
g_{w_{1}, \ldots, w_{n}}(q)=d_{n}(\mathbb{B})^{-\frac{n(n-1)}{2}} q^{-\frac{n(n-1)}{2}} \prod_{1 \leq j<k \leq n}\left(f_{w_{k}}(q)-f_{w_{j}}(q)\right) .
$$

Since $f_{w_{j}}(0)=f(0)$ for every $j=1, \ldots, n$, we get that $g_{w_{1}, \ldots, w_{n}}$ is regular on $\mathbb{B}$. 
Moreover, using the maximum modulus principle as in (18), we can write

$$
\varphi_{n}(r)^{\frac{n(n-1)}{2}}=\max _{w_{1}, \ldots, w_{n} \in \bar{B}} \max _{|q| \leq r}\left|g_{w_{1}, \ldots, w_{n}}(q)\right| ;
$$

hence, we can conclude that $\varphi_{n}(r)$ is increasing in $r$.

In turn, to prove the second part of the statement, we proceed as follows:

$$
\begin{aligned}
\lim _{r \rightarrow 0^{+}} \varphi_{n}(r) & =\lim _{r \rightarrow 0^{+}} \frac{\tilde{d}_{n}(f(r \mathbb{B}))}{d_{n}(\mathbb{B}) r} \\
& =\left.\left.\lim _{r \rightarrow 0^{+}} d_{n}(\mathbb{B})^{-1} r^{-1} \max _{w_{1}, \ldots, w_{n} \in \mathbb{B}} \max _{|q| \leq r}||\right|_{j<k}\left(f_{w_{k}}(q)-f_{w_{j}}(q)\right)\right|^{\frac{2}{n(n-1)}} .
\end{aligned}
$$

We can turn the $*$-product into a usual product with an iterated application of Proposition 2.7 (omitting the points where some factor $f_{w_{k}}(q)-f_{w_{j}}(q)$ vanishes), thus obtaining

$$
\begin{aligned}
& \lim _{r \rightarrow 0^{+}} \varphi_{n}(r) \\
& \quad=\lim _{r \rightarrow 0^{+}} d_{n}(\mathbb{B})^{-1} r^{-1} \max _{w_{1}, \ldots, w_{n} \in \mathbb{B}} \max _{|q| \leq r}\left|\prod_{j<k}\left(f_{w_{k}}\left(T_{j, k}(q)\right)-f_{w_{j}}\left(T_{j, k}(q)\right)\right)\right|^{\frac{2}{n(n-1)}},
\end{aligned}
$$

where for all $j<k, T_{j, k}(q)$ is a suitable quaternion belonging to the same sphere $\operatorname{Re} q+|\operatorname{Im} q| \mathbb{S}$ of $q$. Since for every $j<k$ we have $\left|T_{k, j}(q)\right|=|q|$ if $|q|=r$, using the power series expansion of $f$ we can write

$$
\begin{aligned}
& \lim _{r \rightarrow 0^{+}} \varphi_{n}(r) \\
& \quad=\lim _{r \rightarrow 0^{+}} d_{n}(\mathbb{B})^{-1} \max _{w_{1}, \ldots, w_{n} \in \mathbb{B}} \max _{|q| \leq r} \prod_{j<k}\left|\sum_{n \geq 1}\left(T_{k, j}(q)\right)^{n-1}\left(w_{k}^{n}-w_{j}^{n}\right) a_{n}\right|^{\frac{2}{n(n-1)}} .
\end{aligned}
$$

Since $\varphi_{n}(r)$ is lowerbounded by 0 and it is increasing in $r$ then the limit of $\varphi_{n}(r)$ as $r$ goes to 0 always exists. Proceeding as in the proof of Theorem 3.9, we can find a sequence of points $\left\{q_{m}\right\}_{m \in \mathbb{N}}$ such that $\left|q_{m}\right|=\frac{1}{m}$ for any $m \in \mathbb{N}$, and a sequence of $n$-tuples $\left\{\left(w_{1, m}, \ldots, w_{n, m}\right)\right\}_{m \in \mathbb{N}} \subset \bar{B}^{n}$ converging to some $\left(\hat{w}_{1}, \ldots, \hat{w}_{n}\right) \in \overline{\mathbb{B}}^{n}$ such that

$$
\lim _{m \rightarrow \infty} \varphi_{n}\left(\frac{1}{m}\right)=d_{n}(\mathbb{B})^{-1} \prod_{j<k}\left|\sum_{n \geq 1}\left(T_{k, j}(0)\right)^{n-1}\left(\hat{w}_{k}^{n}-\hat{w}_{j}^{n}\right) a_{n}\right|^{\frac{2}{n(n-1)}} ;
$$

therefore, by Definition 1.4 we obtain

$$
\lim _{m \rightarrow \infty} \varphi_{n}\left(\frac{1}{m}\right)=d_{n}(\mathbb{B})^{-1}\left|a_{1}\right| \prod_{j<k}\left|\left(\hat{w}_{k}-\hat{w}_{j}\right)\right|^{\frac{2}{n(n-1)}} \leq\left|a_{1}\right|=\left|\partial_{c} f(0)\right| .
$$


To prove the opposite inequality, notice that for every choice of $\left\{\tilde{w}_{1}, \ldots, \tilde{w}_{n}\right\} \subset \overline{\mathbb{B}}$,

$$
\begin{aligned}
\lim _{r \rightarrow 0^{+}} \max _{w_{1}, \ldots, w_{n} \in \mathbb{B}} \max _{|q|=r} \prod_{j<k}\left|\sum_{n \geq 1}\left(T_{k, j}(q)\right)^{n-1}\left(w_{k}^{n}-w_{j}^{n}\right) a_{n}\right|^{\frac{2}{n(n-1)}} \\
\quad \geq \lim _{r \rightarrow 0^{+}} \max _{|q|=r} \prod_{j<k}\left|\sum_{n \geq 1}\left(T_{k, j}(q)\right)^{n-1}\left(\tilde{w}_{k}^{n}-\tilde{w}_{j}^{n}\right) a_{n}\right|^{\frac{2}{n(n-1)}},
\end{aligned}
$$

whence

$$
\begin{aligned}
\lim _{r \rightarrow 0^{+}} \max _{w_{1}, \ldots, w_{n} \in \overline{\mathbb{B}}} \max _{|q|=r} \prod_{j<k}\left|\sum_{n \geq 1}\left(T_{k, j}(q)\right)^{n-1}\left(w_{k}^{n}-w_{j}^{n}\right) a_{n}\right|^{\frac{2}{n(n-1)}} \\
\geq \max _{\tilde{w}_{1}, \ldots, \tilde{w}_{n} \in \overline{\mathbb{B}}} \lim _{r \rightarrow 0^{+}} \max _{|q|=r} \prod_{j<k}\left|\sum_{n \geq 1}\left(T_{k, j}(q)\right)^{n-1}\left(\tilde{w}_{k}^{n}-\tilde{w}_{j}^{n}\right) a_{n}\right|^{\frac{2}{n(n-1)}} ;
\end{aligned}
$$

therefore, we conclude that

$$
\lim _{r \rightarrow 0^{+}} \varphi_{n}(r) \geq d_{n}(\mathbb{B})^{-1} \max _{w_{1}, \ldots, w_{n} \in \overline{\mathbb{B}}} \prod_{j<k}\left|\left(w_{j}-w_{k}\right) a_{1}\right|^{\frac{2}{n(n-1)}}=\left|a_{1}\right|=\left|\partial_{c} f(0)\right| .
$$

Using Lemma 4.4, one easily proves the following result:

Theorem 4.5. let $f$ be a regular function on $\mathbb{B}$ such that $\tilde{d}_{n}(f(\mathbb{B}))=d_{n}(\mathbb{B})$. Then

$$
\tilde{d}_{n}(f(r \mathbb{B})) \leq d_{n}(r \mathbb{B})
$$

for every $r \in(0,1)$, and

$$
\left|\partial_{c} f(0)\right| \leq 1 .
$$

We believe that if equality holds in (24) for some $r \in(0,1)$ or in (25) then $f$ is affine, but we were not able to prove this statement. On the one hand, it is easy to see that if $f$ is affine, $f(q)=a+q b$ with $a, b \in \mathbb{H},|b|=1$ then equality holds both in (24) and in (25); on the other hand, we do not yet know, in general, if the converse holds using the notion of regular $n$-diameter (for $n>2$ ).

\section{A 3-diameter version of the Landau-Toeplitz theorem}

In this section we prove a complete 3-diameter version of the Landau-Toeplitz theorem. The proof relies upon the elementary fact that three points lie always in the same plane. For this reason, the 3-diameter of a subset of $\mathbb{A}$, which has dimension 4 , is always attained on a bidimensional section of the set. To compute the 3-diameter of the unit ball of $\mathbb{W}$ we need to recall a preliminary result about what happens in the complex case (for a proof, see [Burckel et al. 2008], for instance). Let $\mathbb{D}$ be the open unit disc of $\mathbb{C}$. 
Lemma 5.1. Given $n$ points $\left\{w_{1}, \ldots, w_{n}\right\} \subset \overline{\mathbb{D}}$, we have

$$
\prod_{1 \leq j<k \leq n}\left|w_{j}-w_{k}\right| \leq n^{\frac{n}{2}}
$$

Equality holds if and only if (after relabeling) $w_{j}=u \alpha^{j}$, where $u \in \mathbb{S}^{1}$ and $\alpha=e^{i 2 \pi / n}$ is an $n$-th root of unity.

Lemma 5.2. Fix any $I \in \mathbb{S}$ and $u \in \partial \mathbb{B}$. The 3-diameter of the unit ball of $\mathbb{W}$ is

$$
d_{3}(\mathbb{B})=\left(\left|\alpha_{2}-\alpha_{1}\right|\left|\alpha_{3}-\alpha_{1}\right|\left|\alpha_{3}-\alpha_{2}\right|\right)^{1 / 3},
$$

where $\alpha_{j}=u e^{I 2 \pi j / 3}$ for $j=1,2,3$.

Proof. The result can be easily proved showing that the 3-diameter is attained on a maximal disc that, without loss of generality, can be chosen to be some $\mathbb{B}_{I}$.

In particular, $d_{3}(\mathbb{B})=d_{3}(\mathbb{D})=\sqrt{3}$. To prove our 3 -diameter version of the Landau-Toeplitz theorem, we introduce an appropriate notion of "slicewise" 3-diameter, inspired by the power series expansion of the regular 3-diameter.

Definition 5.3. Let $f: \mathbb{B} \rightarrow \mathbb{H}$ be a regular function, and let $\sum_{n \geq 0} q^{n} a_{n}$ be its power series expansion. If $a_{N}$ is the first nonvanishing coefficient, let $\hat{f}$ be the function obtained by multiplying $f$ (on the right) by $a_{N}^{-1}\left|a_{N}\right|$ :

$$
\hat{f}(q)=\sum_{n \geq 0} q^{n} a_{n} a_{N}^{-1}\left|a_{N}\right|=\sum_{n \geq 0} q^{n} b_{n} .
$$

This is regular on $\mathbb{B}$ as well. For any $I \in \mathbb{S}$, let $w_{1}, w_{2}, w_{3}$ be points in the closed disc $\overline{\mathbb{B}}_{I}$, and consider the function

$\hat{g}_{w_{1}, w_{2}, w_{3}}(z)=\sum_{n \geq 0} z^{n} \sum_{k=0}^{n} \sum_{j=0}^{k}\left(w_{2}^{j}-w_{1}^{j}\right)\left(w_{3}^{k-j}-w_{1}^{k-j}\right)\left(w_{3}^{n-k}-w_{2}^{n-k}\right) b_{j} b_{k-j} b_{n-k}$,

which is holomorphic in all variables $z, w_{1}, w_{2}, w_{3}$ on $\mathbb{B}_{I}$. We define the slice 3-diameter of $f(r \mathbb{B})$ by

$$
\hat{d}_{3}(f(r \mathbb{B}))=\sup _{I \in \mathbb{S}} \max _{w_{1}, w_{2}, w_{3} \in \overline{\mathbb{B}}_{I}} \max _{z \in r \overline{\mathbb{B}}}\left|\hat{g}_{w_{1}, w_{2}, w_{3}}(z)\right|^{1 / 3},
$$

and the slice 3-diameter of $f(\mathbb{B})$ as the limit

$$
\hat{d}_{3}(f(\mathbb{B}))=\lim _{r \rightarrow 1^{-}} \hat{d}_{3}(f(r \mathbb{B})) .
$$

By the maximum modulus principle (Theorem 2.8), the function $r \mapsto \hat{d}_{3}(f(r \mathbb{B}))$ is increasing; hence, the previous definition is well posed. It is not difficult to prove that $\hat{g}_{w_{1}, w_{2}, w_{3}}(z)$ is continuous as a function of $I$ and of the real and imaginary parts of $z, w_{1}, w_{2}, w_{3}$; hence, the supremum in (26) is actually a maximum. 
Remark 5.4. For any regular function $f: \mathbb{B} \rightarrow \mathbb{H}$, the slice 3-diameter $\hat{d}_{3}(f(\mathbb{B}))$ is the same as the slice 3 -diameter $\hat{d}_{3}((f-f(0))(\mathbb{B}))=\hat{d}_{3}(f(\mathbb{B})-f(0))$. Moreover, it is easy to prove that if the slice 3 -diameter $\hat{d}_{3}(f(\mathbb{B}))$ vanishes then $\mathrm{f}$ is constant.

Lemma 5.5. In analogy with what happens in the regular $n$-diameter case, let $f$ be a regular function on $\mathbb{B}$, and for $r \in(0,1)$ let $\hat{\varphi}_{3}(r)$ be the ratio defined as

$$
\hat{\varphi}_{3}(r)=\frac{\hat{d}_{3}(f(r \mathbb{B}))}{d_{3}(r \mathbb{B})}=\frac{\hat{d}_{3}(f(r \mathbb{B}))}{d_{3}(\mathbb{B}) r} .
$$

Then $\hat{\varphi}_{3}(r)$ is increasing in $r$ and

$$
\lim _{r \rightarrow 0^{+}} \hat{\varphi}_{3}(r)=\left|\partial_{c} f(0)\right| .
$$

Proof. One proves that

$$
\hat{\varphi}_{3}(r)^{3}=d_{3}(\mathbb{B})^{-3} \max _{I \in \mathbb{S}} \max _{w_{1}, w_{2}, w_{3} \in \overline{\mathbb{B}}_{I}} \max _{z \in r \overline{\mathbb{B}}_{I}}\left|z^{-3} \hat{g}_{w_{1}, w_{2}, w_{3}}(z)\right|,
$$

(see Definition 5.3) and uses the technique of the proof of Lemma 4.4 on each slice.

The fundamental tool to prove the "equality case" is the following:

Theorem 5.6. Let $f$ be a regular function on $\mathbb{B}$ and for $r \in(0,1)$, let

$$
\hat{\varphi}_{3}(r)=\frac{\hat{d}_{3}(f(r \mathbb{B}))}{d_{3}(\mathbb{B}) r} .
$$

Then $\hat{\varphi}_{3}(r)$ is strictly increasing in $r$ except if $f$ is a constant or affine function; that is, if $f(q)=a+q b$ with $a, b \in \mathbb{U}$.

Proof. Thanks to Remark 5.4 we can suppose $f(0)=0$. Since $\hat{\varphi}_{3}(r)$ is increasing for $r \in(0,1)$, if it is not strictly increasing then there exist $s, t, 0<s<t<1$ such that $\hat{\varphi}_{3}$ is constant on $[s, t]$. We will show that this yields that $\hat{\varphi}_{3}$ is constant on $(0, t]$. Let $I \in \mathbb{S}$ and $w_{1}, w_{2}, w_{3} \in \overline{\mathbb{B}}_{I}$ be such that

$$
\hat{\varphi}_{3}(s)^{3}=d_{3}(\mathbb{B})^{-3} \max _{z \in s \overline{\mathbb{B}}_{I}}\left|z^{-3} \hat{g}_{w_{1}, w_{2}, w_{3}}(z)\right| .
$$

For $r \in[s, t]$, we have $\hat{\varphi}_{3}(r)=\hat{\varphi}_{3}(s)$ and by the choice of $w_{1}, w_{2}, w_{3}$,

$$
\begin{aligned}
\hat{\varphi}_{3}(r)^{3} & \geq d_{3}(\mathbb{B})^{-3} \max _{z \in r \overline{\mathbb{B}}_{I}}\left|z^{-3} \hat{g}_{w_{1}, w_{2}, w_{3}}(z)\right| \\
& \geq d_{3}(\mathbb{B})^{-3} \max _{z \in s \overline{\mathbb{B}}_{I}}\left|z^{-3} \hat{g}_{w_{1}, w_{2}, w_{3}}(z)\right|=\hat{\varphi}_{3}(s)^{3} ;
\end{aligned}
$$

hence, by the maximum modulus principle (see Theorem 2.8), we get that the function $z \mapsto z^{-3} \hat{g}_{w_{1}, w_{2}, w_{3}}(z)$ is constant on $\mathbb{B}_{I}$. If we now consider $r \in(0, s)$ 
then $\hat{\varphi}_{3}(r) \leq \hat{\varphi}_{3}(s)$, and

$$
\begin{aligned}
\hat{\varphi}_{3}(r)^{3} & \geq d_{3}(\mathbb{B})^{-3} \max _{z \in r \overline{\mathbb{B}}_{I}}\left|z^{-3} \hat{g}_{w_{1}, w_{2}, w_{3}}(z)\right| \\
& =d_{3}(\mathbb{B})^{-3} \max _{z \in s \overline{\mathbb{B}}_{I}}\left|z^{-3} \hat{g}_{w_{1}, w_{2}, w_{3}}(z)\right|=\hat{\varphi}_{3}(s)^{3} ;
\end{aligned}
$$

hence, $\hat{\varphi}_{3}(r)=\hat{\varphi}_{3}(s)$ for all $r \in(0, t]$. Thanks to Lemma 5.5, we obtain

$$
\hat{\varphi}_{3}(r) \equiv \lim _{r \rightarrow 0^{+}} \hat{\varphi}_{3}(r)=\left|\partial_{c} f(0)\right|=\left|a_{1}\right| \quad \text { for } r \in[0, t] .
$$

Recalling Remark 5.4, we get that either $f$ is constant or $a_{1}=\partial_{c} f(0) \neq 0$. Let us suppose that $f$ is not constant (so that $b_{n}=a_{n} a_{1}^{-1}\left|a_{1}\right|$ for any $n \in \mathbb{N}$ ). Recalling the definition of $\hat{g}_{w_{1}, w_{2}, w_{3}}(z)$, and since the (constant) function $z \mapsto z^{-3} \hat{g}_{w_{1}, w_{2}, w_{3}}(z)$ is equal to its limit at 0 , we have

$$
\begin{aligned}
& \left|a_{1}\right|^{3} \\
& =\hat{\varphi}_{3}^{3}(r) \\
& =\frac{1}{d_{3}(\mathbb{B})^{3}}\left|\sum_{n \geq 3} z^{n-3} \sum_{k=0}^{n} \sum_{j=0}^{k}\left(w_{2}^{j}-w_{1}^{j}\right)\left(w_{3}^{k-j}-w_{1}^{k-j}\right)\left(w_{3}^{n-k}-w_{2}^{n-k}\right) b_{j} b_{k-j} b_{n-k}\right| \\
& =\frac{1}{d_{3}(\mathbb{B})^{3}}\left|\left(w_{2}-w_{1}\right)\left(w_{3}-w_{1}\right)\left(w_{3}-w_{2}\right) b_{1}^{3}\right|
\end{aligned}
$$

for any $z \in \mathbb{B}_{I}$; therefore, thanks to Lemma 5.2, without loss of generality we can suppose that $w_{1}=1, w_{2}, w_{3}$ are cube roots of unity in $L_{I}$. Now let $J$ be an imaginary unit, $J \neq I$, and consider $v_{1}, v_{2}, v_{3}$ cube roots of unity in $L_{J}$. Then, for any $r \in[0, t]$,

$$
\begin{aligned}
\left|a_{1}\right| & =\hat{\varphi}_{3}(r)=d_{3}(\mathbb{B})^{-1} \max _{I \in \mathbb{S}} \max _{w_{1}, w_{2}, w_{3} \in \overline{\mathbb{B}}_{I}} \max _{z \in r \overline{\mathbb{B}}_{I}}\left|z^{-3} \hat{g}_{w_{1}, w_{2}, w_{3}}(z)\right|^{1 / 3} \\
& \geq d_{3}(\mathbb{B})^{-1} \max _{z \in r \overline{\mathbb{B}}_{J}}\left|z^{-3} \hat{g}_{v_{1}, v_{2}, v_{3}}(z)\right|^{1 / 3} \geq d_{3}(\mathbb{B})^{-1}\left|z^{-3} \hat{g}_{v_{1}, v_{2}, v_{3}}(z)\right|_{z=0}^{1 / 3}=\left|a_{1}\right| ;
\end{aligned}
$$

therefore, for any $J \in \mathbb{S}$, if $v_{1}, v_{2}, v_{3}$ are cube roots of unity in $L_{J}$, the function $z \mapsto z^{-3} \hat{g}_{v_{1}, v_{2}, v_{3}}(z) \equiv c_{J}$ is constant on $\mathbb{B}_{J}$. Notice that $\left|c_{J}\right|$ does not depend on $J \in \mathbb{S}$. Now let $I$ be an imaginary unit in $\mathbb{S}$, fix $z \in t \mathbb{B}_{I}$ with $|z|=r$, and let $w_{1}=1, w_{2}, w_{3}$ be cube roots of unity in $L_{I}$. Consider the function defined for $\zeta \in \mathbb{B}_{I}$ by

$$
\begin{aligned}
h_{z}^{I}(\zeta) & =z^{-3} \hat{g}_{\zeta, w_{2}, w_{3}}(z) \\
& =\sum_{n \geq 3} z^{n-3} \sum_{k=0}^{n} \sum_{j=0}^{k}\left(w_{2}^{j}-\zeta^{j}\right)\left(w_{3}^{k-j}-\zeta^{k-j}\right)\left(w_{3}^{n-k}-w_{2}^{n-k}\right) b_{j} b_{k-j} b_{n-k} .
\end{aligned}
$$


By construction, $\zeta \mapsto h_{z}^{I}(\zeta)$ is holomorphic on a neighborhood of $\overline{\mathbb{B}}_{I}$, and

$$
\left|h_{z}^{I}(\zeta)\right| \leq \hat{\varphi}_{3}(r)^{3} d_{3}(\mathbb{B})^{3}=\left|a_{1}\right|^{3} d_{3}(\mathbb{B})^{3} .
$$

Its value at $\zeta=1$ is

$$
h_{z}^{I}(1)=z^{-3} g_{1, w_{2}, w_{3}}(z)=\left(w_{2}-1\right)\left(w_{3}-1\right)\left(w_{3}-w_{2}\right) b_{1}^{3}=-3 \sqrt{3} I\left|a_{1}\right|^{3} .
$$

Then the function

$$
\zeta \mapsto h_{z}^{I}(\zeta)\left(h_{z}^{I}(1)\right)^{-1}=h_{z}^{I}(\zeta) I(3 \sqrt{3})^{-1}\left|a_{1}\right|^{-3}
$$

fixes the point $\zeta=1$ and maps the closed unit disc $\overline{\mathbb{B}}_{I}$ to itself; in fact,

$$
\begin{aligned}
\left.\left|h_{z}^{I}(\zeta) I(3 \sqrt{3})^{-1}\right| a_{1}\right|^{-3} \mid & =\left|h_{z}^{I}(\zeta)\right|(3 \sqrt{3})^{-1}\left|a_{1}\right|^{-3} \\
& \leq\left|a_{1}\right|^{3} d_{3}(\mathbb{B})^{3}(3 \sqrt{3})^{-1}\left|a_{1}\right|^{-3}=1 .
\end{aligned}
$$

We can therefore apply Proposition 3.2 and we get

$$
\operatorname{Im}_{I}\left(\left.\frac{\partial}{\partial \zeta}\right|_{\zeta=1} h_{z}^{I}(\zeta) I(3 \sqrt{3})^{-1}\left|a_{1}\right|^{-3}\right)=0 ; \quad \text { that is, } \quad \operatorname{Re}\left(\frac{\partial}{\partial \zeta} h_{z}^{I}(1)\right)=0 .
$$

Doing the same construction for any $J \in \mathbb{S}$, we get that

$$
\operatorname{Re}\left(\frac{\partial}{\partial \zeta} h_{z}^{J}(1)\right)=0
$$

for any fixed $z \in t \mathbb{B}_{J}$. An easy computation shows that

$$
\begin{aligned}
& \frac{\partial}{\partial \zeta} h_{z}^{I}(1) \\
& =-\sum_{n \geq 3} z^{n-3} \sum_{k=2}^{n-1} \sum_{j=1}^{k-1}\left(j\left(w_{3}^{k-j}-1\right)+(k-j)\left(w_{2}^{j}-1\right)\right)\left(w_{3}^{n-k}-w_{2}^{n-k}\right) b_{j} b_{k-j} b_{n-k} .
\end{aligned}
$$

Thanks to the uniform convergence of the series expansion and since (27) holds for any $z \in t \mathbb{B}_{I}$, we get that the real part of each coefficient must vanish. Namely, for any $n \in \mathbb{N}, n \geq 3$,

$\operatorname{Re}\left(\sum_{k=2}^{n-1} \sum_{j=1}^{k-1}\left(j\left(w_{3}^{k-j}-1\right)+(k-j)\left(w_{2}^{j}-1\right)\right)\left(w_{3}^{n-k}-w_{2}^{n-k}\right) b_{j} b_{k-j} b_{n-k}\right)=0$.

That this is true for any $I \in \mathbb{S}$ will allow us to show that $b_{n}=a_{n}\left(a_{1}^{-1}\left|a_{1}\right|\right)$ is real for any $n \in \mathbb{N}$. We do this by induction. The first step is trivial; $b_{0}=0$ and

$$
b_{1}=a_{1}\left(a_{1}^{-1}\left|a_{1}\right|\right)=\left|a_{1}\right| .
$$


Suppose then that $b_{1}, \ldots, b_{s-1}$ are real numbers. The first coefficient of the series expansion of $(\partial / \partial \zeta) h_{z}^{I}(1)$ that contains $b_{s}$ is the one for which $n=s+2$, and which has to satisfy

(28)

$\operatorname{Re} \sum_{k=2}^{s+1} \sum_{j=1}^{k-1}\left(j\left(w_{3}^{k-j}-1\right)+(k-j)\left(w_{2}^{j}-1\right)\right)\left(w_{3}^{s+2-k}-w_{2}^{s+2-k}\right) b_{j} b_{k-j} b_{s+2-k}=0$.

Three terms in this sum involve $b_{s}$ : those with $(k, j)=(s+1, s),(s+1,1),(2,1)$. After some manipulations, their sum is seen to equal

$$
\left.\left(\sqrt{3} I\left(3 s+2-\left(w_{2}^{s}+w_{3}^{s}\right)\right)-3\left(w_{2}^{s}-w_{3}^{s}\right)\right)\right)\left|a_{1}\right|^{2} b_{s} .
$$

hence, we can split the sum in (28) into

$$
\left.\left(\sqrt{3} I\left(3 s+2-\left(w_{2}^{s}+w_{3}^{s}\right)\right)-3\left(w_{2}^{s}-w_{3}^{s}\right)\right)\right)\left|a_{1}\right|^{2} b_{s}+\Sigma_{1}+\Sigma_{2},
$$

where

$$
\begin{aligned}
& \Sigma_{1}=\sum_{j=2}^{s-1}\left(j\left(w_{3}^{s+1-j}-1\right)+(s+1-j)\left(w_{2}^{j}-1\right)\right)\left(w_{3}-w_{2}\right) b_{j} b_{s+1-j} b_{1}, \\
& \Sigma_{2}=\sum_{k=3}^{s} \sum_{j=1}^{k-1}\left(j\left(w_{3}^{k-j}-1\right)+(k-j)\left(w_{2}^{j}-1\right)\right)\left(w_{3}^{s+2-k}-w_{2}^{s+2-k}\right) b_{j} b_{k-j} b_{s+2-k} .
\end{aligned}
$$

We claim that $\operatorname{Re} \Sigma_{1}$ and $\operatorname{Re} \Sigma_{2}$ vanish. Indeed, if $s$ is even, we can express $\Sigma_{1}$ as

$$
\sum_{j=2}^{s / 2}\left(j\left(w_{3}^{s+1-j}+w_{2}^{s+1-j}-2\right)+(s+1-j)\left(w_{2}^{j}+w_{3}^{j}-2\right)\right)\left(w_{3}-w_{2}\right) b_{j} b_{s+1-j} b_{1} \text {. }
$$

The real part of each summand vanishes because $w_{2}^{n}+w_{3}^{n} \in \mathbb{R}$ and $w_{2}^{n}-w_{3}^{n} \in I \mathbb{R}$ for any $n \in \mathbb{N}$, while $b_{n} \in \mathbb{R}$ for any $n=1, \ldots, s-1$. This shows that $\operatorname{Re} \Sigma_{1}=0$ when $s$ is even. The proofs for $\Sigma_{1}$ with $s$ odd and for $\Sigma_{2}$ are similar.

We have reduced (28) to

$$
\left.\operatorname{Re}\left(\left(\sqrt{3} I\left(3 s+2-\left(w_{2}^{s}+w_{3}^{s}\right)\right)-3\left(w_{2}^{s}-w_{3}^{s}\right)\right)\right)\left|a_{1}\right|^{2} b_{s}\right)=0 .
$$

Therefore, for any $s \in \mathbb{N}$, there exists $\alpha_{s} \in \mathbb{R}$ such that $\operatorname{Re}\left(\alpha_{s} I b_{s}\right)=\alpha_{s} \operatorname{Re}\left(I b_{s}\right)=$ $\operatorname{Im}_{I}\left(b_{s}\right)=0$ for all $I \in \mathbb{S}$; hence, we get $b_{s} \in \mathbb{R}$ for all $s$. Recalling that the $b_{n}$ are the coefficients of the power series of $\hat{f}$, we get that $\hat{f}\left(\mathbb{B}_{I}\right) \subseteq L_{I}$ for all $I \in \mathbb{S}$; hence, $\hat{f}$ is complex holomorphic on each slice. 
We now claim that for any $r \in(0, t]$ the slice 3-diameter of $f(r \mathbb{B})$ coincides with the usual 3-diameter of $\hat{f}(r \mathbb{B})$. Indeed, for any $I \in \mathbb{S}$, we have $\hat{g}_{w_{1}, w_{2}, w_{3}}(z)$

$$
\begin{gathered}
=\sum_{n \geq 0} z^{n} \sum_{k=0}^{n} \sum_{j=0}^{k}\left(w_{2}^{j}-w_{1}^{j}\right)\left(w_{3}^{k-j}-w_{1}^{k-j}\right)\left(w_{3}^{n-k}-w_{2}^{n-k}\right) b_{j} b_{k-j} b_{n-k} \\
=\left(\sum_{n \geq 0}\left(\left(z w_{2}\right)^{n}-\left(z w_{1}\right)^{n}\right) b_{n}\right)\left(\sum_{n \geq 0}\left(\left(z w_{3}\right)^{n}-\left(z w_{1}\right)^{n}\right) b_{n}\right) \\
\times\left(\sum_{n \geq 0}\left(\left(z w_{3}\right)^{n}-\left(z w_{2}\right)^{n}\right) b_{n}\right),
\end{gathered}
$$

which is to say

$$
\hat{g}_{w_{1}, w_{2}, w_{3}}(z)=\left(\hat{f}\left(z w_{2}\right)-\hat{f}\left(z w_{1}\right)\right)\left(\hat{f}\left(z w_{3}\right)-\hat{f}\left(z w_{1}\right)\right)\left(\hat{f}\left(z w_{3}\right)-\hat{f}\left(z w_{2}\right)\right) .
$$

For each side we take the absolute value, the third root and the maximum over $z \in r \overline{\mathbb{B}}_{I}$ and $w_{1}, w_{2}, w_{3} \in \overline{\mathbb{B}}_{I}$, to obtain $\hat{d}_{3}(f(r \mathbb{B}))=d_{3}\left(\hat{f}\left(r \mathbb{B}_{I}\right)\right)$, as desired.

Thanks to the complex $n$-diameter version of the Landau-Toeplitz theorem [Burckel et al. 2008] we conclude that $\hat{f}$ is an affine function,

$$
\hat{f}(q)=b_{0}+q b_{1}=a_{0} a_{1}^{-1}\left|a_{1}\right|+q\left|a_{1}\right| .
$$

Hence $f$ is affine as well: $f(q)=a_{0}+q a_{1}$.

Theorem 5.7 (Landau-Toeplitz theorem for the slice 3-diameter). Let $f$ be a regular function on $\mathbb{B}$ such that $\hat{d}_{3} f(\mathbb{B})=d_{3}(\mathbb{B})$. Then

$$
\hat{d}_{3}(f(r \mathbb{B})) \leq d_{3}(r \mathbb{B})
$$

for every $r \in(0,1)$, and

$$
\left|\partial_{c} f(0)\right| \leq 1
$$

Equality holds in (30) for some $r \in(0,1)$, or in (31), if and only if $f$ is an affine function $f(q)=a+q b$ with $a, b \in \mathbb{H}$ and $|b|=1$.

Proof. By Lemma 5.5, both inequalities hold true. For the equality case, if $f(q)=$ $a+q b$ with $a, b \in \mathbb{U},|b|=1$, it is easy to see that equality holds in both statements; otherwise, if equality holds in (30) or in (31) then $\hat{\varphi}_{3}(r)$ defined in Lemma 5.5 is not strictly increasing. Theorem 5.6 then implies that $f$ is an affine function. Since $\hat{d}_{3}\left((f(\mathbb{B}))=d_{3}(\mathbb{B})\right.$, the coefficient of the first degree term of $f$ has unitary modulus. 
Notice that the notion of the slice 3-diameter does not make sense for $n \geq 4$. Moreover, the $n$-diameter of $\mathbb{B}$, when $n \geq 4$, is not anymore attained at points that lie on the same plane $L_{I}$; in fact, the following result holds true:

Proposition 5.8. For all $I \in \mathbb{S}$ the inequality $d_{4}(\mathbb{B})>d_{4}\left(\mathbb{B}_{I}\right)$ holds.

Proof. The proof follows from the direct computation of the 4-diameter of a maximal tetrahedron contained in $\mathbb{B}$.

The proof of Theorem 5.6 heavily relies upon the fact that both the 3-diameter of $\mathbb{B}$ and the slice 3-diameter of $f(\mathbb{B})$ are attained at a complete set of cube roots of unity lying on a same plane $L_{I}$. We have no alternative proof to use when $n \geq 4$.

\section{References}

[Abate 1989] M. Abate, Iteration theory of holomorphic maps on taut manifolds, Mediterranean Press, Rende, 1989. MR 92i:32032 Zbl 0747.32002

[Burckel 1979] R. B. Burckel, An introduction to classical complex analysis, 1, Pure and Applied Mathematics 82, Academic Press, New York, 1979. MR 81d:30001 Zbl 0434.30002

[Burckel et al. 2006] R. B. Burckel, D. E. Marshall, and P. Poggi-Corradini, "On a theorem of Landau and Toeplitz", preprint, 2006. arXiv math/0603579v1

[Burckel et al. 2008] R. B. Burckel, D. E. Marshall, D. Minda, P. Poggi-Corradini, and T. J. Ransford, "Area, capacity and diameter versions of Schwarz's lemma", Conform. Geom. Dyn. 12 (2008), 133-152. MR 2010j:30050 Zbl 1233.30016

[Colombo et al. 2009] F. Colombo, G. Gentili, I. Sabadini, and D. Struppa, "Extension results for slice regular functions of a quaternionic variable", Adv. Math. 222:5 (2009), 1793-1808. MR 2010j:30102 Zbl 1179.30052

[Cullen 1965] C. G. Cullen, "An integral theorem for analytic intrinsic functions on quaternions", Duke Math. J. 32 (1965), 139-148. MR 30 \#3227 Zbl 0173.09001

[Della Rocchetta et al. 2012] C. Della Rocchetta, G. Gentili, and G. Sarfatti, "The Bohr theorem for slice regular functions", Math. Nachr. 285:17-18 (2012), 2093-2105. MR 3002603 Zbl 1257.30053

[Della Rocchetta et al. 2013] C. Della Rocchetta, G. Gentili, and G. Sarfatti, "A Bloch-Landau theorem for slice regular functions", pp. 55-74 in Advances in hypercomplex analysis, edited by G. Gentili et al., Springer-INdAM series 1, Springer, Milan, 2013. Zbl 06148976

[Gardner 2006] R. J. Gardner, Geometric tomography, 2nd ed., Encyclopedia of Mathematics and its Applications 58, Cambridge University Press, 2006. MR 2007i:52010 Zbl 1102.52002

[Gentili and Struppa 2006] G. Gentili and D. C. Struppa, "A new approach to Cullen-regular functions of a quaternionic variable", C. R. Math. Acad. Sci. Paris 342:10 (2006), 741-744. MR 2006m:30095 Zbl 1105.30037

[Gentili and Struppa 2007] G. Gentili and D. C. Struppa, "A new theory of regular functions of a quaternionic variable”, Adv. Math. 216:1 (2007), 279-301. MR 2008h:30052 Zbl 1124.30015

[Gentili et al. 2013] G. Gentili, C. Stoppato, and D. C. Struppa, Regular functions of a quaternionic variable, Springer, Heidelberg, 2013. MR 3013643 Zbl 06090418

[Lachand-Robert and Oudet 2007] T. Lachand-Robert and É. Oudet, "Bodies of constant width in arbitrary dimension”, Math. Nachr. 280:7 (2007), 740-750. MR 2008d:52002 Zbl 1121.52009 
[Landau and Toeplitz 1907] E. Landau and O. Toeplitz, "Über die größte Schwankung einer analytischen Funktion in einem Kreise”, Arch. Math. Phys. (3) 11 (1907), 302-307. JFM 38.0433.02

[Sarfatti 2013] G. Sarfatti, Elements of function theory in the unit ball of quaternions, Ph.D. thesis, Università di Firenze, 2013, www.students.math.unifi.it/users/sarfatti/PhDGiulia.pdf.

[Solynin 2008] A. Y. Solynin, "A Schwarz lemma for meromorphic functions and estimates for the hyperbolic metric", Proc. Amer. Math. Soc. 136:9 (2008), 3133-3143. MR 2009c:30064 Zbl 1151.30017

Received June 28, 2011. Revised April 14, 2013.

GRAZIANO GENTILI

Dipartimento di Matematica e InFormatica "U. Dini”

UNIVERSITÀ DI FIRENZE

Viale MORGAGNi 67/A

50134 FIRENZE

ITALY

gentili@math.unifi.it

GiUlia SARFATTI

Dipartimento di MATEMATICA E INFORMATICA “U. Dini” UNIVERSITÀ DI FIRENZE

Viale Morgagni 67/A

50134 FIRENZE

ITALY

sarfatti@math.unifi.it 


\title{
PACIFIC JOURNAL OF MATHEMATICS
}

\author{
msp.org/pjm
}

Founded in 1951 by E. F. Beckenbach (1906-1982) and F. Wolf (1904-1989)

\section{EDITORS}

V. S. Varadarajan (Managing Editor)

Department of Mathematics

University of California

Los Angeles, CA 90095-1555

pacific@math.ucla.edu

Paul Balmer

Department of Mathematics

University of California

Los Angeles, CA 90095-1555

balmer@math.ucla.edu

Daryl Cooper

Department of Mathematics

University of California

Santa Barbara, CA 93106-3080 cooper@math.ucsb.edu

Jiang-Hua $\mathrm{Lu}$

Department of Mathematics

Pokfulam Rd., Hong Kong jhlu@maths.hku.hk
The University of Hong Kong

Don Blasius

Department of Mathematics University of California

Los Angeles, CA 90095-1555

blasius@math.ucla.edu

Robert Finn

Department of Mathematics Stanford University

Stanford, CA 94305-2125

finn@math.stanford.edu

Sorin Popa

Department of Mathematics

University of California

Los Angeles, CA 90095-1555 popa@math.ucla.edu

Paul Yang

Department of Mathematics Princeton University

Princeton NJ 08544-1000

yang@math.princeton.edu

\section{PRODUCTION}

Silvio Levy, Scientific Editor, production@msp.org

\section{SUPPORTING INSTITUTIONS}

ACADEMIA SINICA, TAIPEI

CALIFORNIA INST. OF TECHNOLOGY

INST. DE MATEMÁTICA PURA E APLICADA

KEIO UNIVERSITY

MATH. SCIENCES RESEARCH INSTITUTE

NEW MEXICO STATE UNIV.

OREGON STATE UNIV.

\author{
STANFORD UNIVERSITY \\ UNIV. OF BRITISH COLUMBIA \\ UNIV. OF CALIFORNIA, BERKELEY \\ UNIV. OF CALIFORNIA, DAVIS \\ UNIV. OF CALIFORNIA, LOS ANGELES \\ UNIV. OF CALIFORNIA, RIVERSIDE \\ UNIV. OF CALIFORNIA, SAN DIEGO \\ UNIV. OF CALIF., SANTA BARBARA
}

\author{
Vyjayanthi Chari \\ Department of Mathematics \\ University of California \\ Riverside, CA 92521-0135 \\ chari@math.ucr.edu \\ Kefeng Liu \\ Department of Mathematics \\ University of California \\ Los Angeles, CA 90095-1555 \\ liu@math.ucla.edu \\ Jie Qing \\ Department of Mathematics \\ University of California \\ Santa Cruz, CA 95064 \\ qing@cats.ucsc.edu
}

These supporting institutions contribute to the cost of publication of this Journal, but they are not owners or publishers and have no responsibility for its contents or policies.

See inside back cover or msp.org/pjm for submission instructions.

The subscription price for 2013 is US \$400/year for the electronic version, and \$485/year for print and electronic.

Subscriptions, requests for back issues and changes of subscribers address should be sent to Pacific Journal of Mathematics, P.O. Box 4163, Berkeley, CA 94704-0163, U.S.A. The Pacific Journal of Mathematics is indexed by Mathematical Reviews, Zentralblatt MATH, PASCAL CNRS Index, Referativnyi Zhurnal, Current Mathematical Publications and the Science Citation Index.

The Pacific Journal of Mathematics (ISSN 0030-8730) at the University of California, c/o Department of Mathematics, 798 Evans Hall \#3840, Berkeley, CA 94720-3840, is published twelve times a year. Periodical rate postage paid at Berkeley, CA 94704, and additional mailing offices. POSTMASTER: send address changes to Pacific Journal of Mathematics, P.O. Box 4163, Berkeley, CA 94704-0163.

PJM peer review and production are managed by EditFLOW ${ }^{\circledR}$ from Mathematical Sciences Publishers.

\section{PUBLISHED BY}

mathematical sciences publishers

nonprofit scientific publishing

http://msp.org/

(C) 2013 Mathematical Sciences Publishers 


\section{PACIFIC JOURNAL OF MATHEMATICS}

Volume $265 \quad$ No. $2 \quad$ October 2013

Singularity removability at branch points for Willmore surfaces

YANN BERNARD and TRISTAN RIVIÈRE

On Bach flat warped product Einstein manifolds

QIANG CHEN and CHENXU HE

On plane sextics with double singular points

Alex DeGTyareV

A computational approach to the Kostant-Sekiguchi correspondence

HEIKo DiETRICH and WILLEM A. DE GRAAF

Landau-Toeplitz theorems for slice regular functions over quaternions

GRAZIANO GENTILI and GiUlia SARFATTI

On surgery curves for genus-one slice knots

PATRICK M. GILMER and CHARLES LIVINGSTON

Characterizing abelian varieties by the reduction of the Mordell-Weil group

Chris Hall and Antonella Perucca

Variation of complex structures and the stability of Kähler-Ricci

Solitons

StUART J. HALl and ThOMAS MURPHY

On crossed homomorphisms of the volume preserving diffeomorphism groups

RYOJI KASAGAWA

Regularity at the boundary and tangential regularity of solutions of the Cauchy-Riemann system

TRAN VU KHANH and GIUSEPPE ZAMPIERI

On the Steinberg character of a semisimple $p$-adic group 